\title{
Prebiotic synthesis of amide bonds and implications for origin of life
}

ZIMING YANG, YIJU LIAO AND ALEXANDRIA ASPIN

Oakland University

Presenting Author: zimingyang@oakland.edu

Prebiotic synthesis of organic compounds is critical for forming large and complex biomolecules such as peptides and proteins that are required for life. Hydrothermal systems provide unique reaction conditions for prebiotic synthesis, and they are considered one of the most likely habitable environments for early life. Hydrothermal activities are not only observed on Earth but are also believed to exist on icy worlds including Saturn's moon Enceladus. Understanding the prebiotic chemistry and organic reaction pathways in hydrothermal systems is thus important to uncover key factors and potential driving forces for the origin-of-life processes on Earth. In a recent study, we observed a feasible hydrothermal pathway for amide bond synthesis through a direct condensation between amines and carboxylic acids. As building blocks for life, peptides and proteins require the formation of peptide bond from amino acids, with amide structures as the backbone. This relatively facile amide bond formation from simple organic compounds suggests a possible pathway for prebiotic synthesis in hydrothermal environments. To obtain further understanding of this reaction, we examined the effects of environmental factors such as $\mathrm{pH}$, metal ions, and minerals on the amide hydrothermal formation. We find that the amide bond synthesis could be more influenced by fluid chemistry (e.g., $\mathrm{pH}$, ionic species) than the mineral surface. These findings suggest that prebiotic synthesis of peptides and biomolecule precursors in hydrothermal fluids would require well-constrained geochemical settings, which could also be important for predicting the likelihood of prebiotic synthesis and origin of life in other planetary environments. 